\title{
SIMULATION OF DYNAMIC FRACTURE OF CONCRETE WITH DAMAGED VISCOELASTICITY AND RETARDED DAMAGE
}

\author{
U. HÄUSSLER-COMBE* ${ }^{*}$, E. PANTEKI ${ }^{\S}$, M. QUAST ${ }^{\dagger}$ AND T. KÜHN ${ }^{\ddagger}$ \\ Technische Universität Dresden \\ Dresden, Germany \\ e-mail: \\ *ulrich.haeussler-combe@tu-dresden.de \\ §evmorfia.panteki@tu-dresden.de \\ †matthias.quast@tu-dresden.de \\ †tino.kuehn@tu-dresden.de
}

Key words: Concrete, Retarded Damage, Damaged Viscoelasticity, Strain-Rate Effect, Biaxial SplitHopkinson Bar

\begin{abstract}
Many aspects of the strain-rate effect observed for quasi-brittle materials like concrete are still under discussion. A triaxial material model is described covering the physical mechanisms presumably causing the strain-rate effect. Its basic effects on stress-strain behavior are shown with a homogeneous one-element setup. Furthermore, it is applied for the simulation of a biaxial Split-Hopkinson-Bar setup and validated against corresponding experimental results. The analysis of simulation results shows a complex behavior highly variable in space and time.
\end{abstract}

\section{INTRODUCTION}

The behavior of concrete exposed to high strain-rates still has many unexplained aspects. Strain-rates occur in a range from roughly $10^{-5} \mathrm{~s}^{-1}$ for quasistatic conditions up to $10^{3} \mathrm{~s}^{-1}$ caused by, e.g., contact explosions. Experimental data indicate a considerable increase of strength for compression [1] and especially for tension [9], see Fig. 1] for the dynamic increase factors for uniaxial strength (DIF). The course of tensile DIF-values may be approximated by two linear branches in a semi-logarithmic scale. According to the current state of knowledge each branch may be connected to a particular physical mechanism regarding strength increase or the strain-rate effect. The flat branch is related to water which is more or less physically bound in the capillary systems of mortar. It is moved due to deformations and exhibits a higher resistance under high strain-rate conditions [13]. This phenomenon is overlaid by re- tarded damage related to the steeper branch.

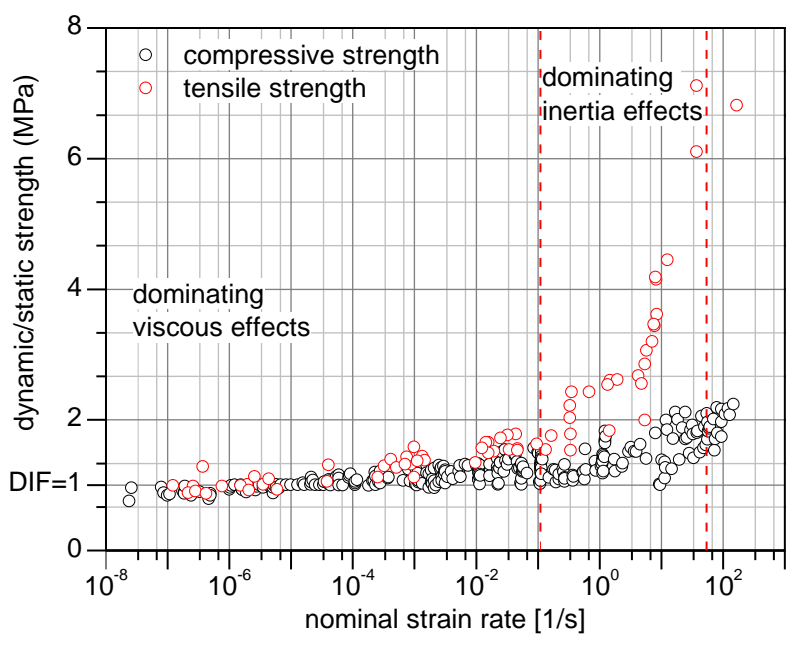

Figure 1: Experimental DIF-data concrete.

Damage results from crack formation. Such crack formations cannot spread arbitrarily fast [12], [4]. This is observed for macro cracks 
with limited crack speed which is lower than the Rayleigh wave speed. The effect basically applies also for the formation of micro cracks. Thus, damage is retarded under high strain-rate conditions compared to the quasistatic conditions. The paper describes a triaxial material model covering both physical aspects by damaged viscoelasticity and retarded damage. These contributions are connected to the strain-rate and base upon an isotropic damage law for the quasistatic behavior of concrete. The validity of this approach is discussed against experimental investigations with a biaxial Split-Hopkinson-Bar (SHB) which allows for the experimental investigation of biaxial stress states. The paper is organized as follows: Section 2 develops the material model. Section 3 describes its application to the simulation of a spatially homogeneous behavior with an one-element setup. The experimental setup of the biaxial SHB is given in Section 4. The experimental results are compared to simulation results in Section 5. Numerical simulation allows for a comprehensive evaluation of the complex specimen behavior, which is highly variable in space and time. Some conclusions are given in Section 6 .

\section{MATERIAL MODEL}

\subsection{Decompositions}

Stress $\sigma$ and strain $\epsilon$ are decomposed in their volumetric and deviatoric parts with

$$
\begin{gathered}
\boldsymbol{\sigma}=\boldsymbol{\sigma}^{v o l}+\boldsymbol{\sigma}^{d e v} \\
\boldsymbol{\sigma}^{v o l}=\boldsymbol{I}^{v o l} \cdot \boldsymbol{\sigma}, \quad \boldsymbol{\sigma}^{d e v}=\boldsymbol{I}^{d e v} \cdot \boldsymbol{\sigma} \\
\boldsymbol{\epsilon}=\boldsymbol{\epsilon}^{v o l}+\boldsymbol{\epsilon}^{d e v} \\
\boldsymbol{\epsilon}^{v o l}=\boldsymbol{I}^{v o l} \cdot \boldsymbol{\epsilon}, \quad \boldsymbol{\epsilon}^{d e v}=\boldsymbol{I}^{d e v} \cdot \boldsymbol{\epsilon}
\end{gathered}
$$

whereby tensor components are also used for the strain $\epsilon$. A vector notation is implicitely used for stresses and strains in the following, whereby matrices are employed for quantities like higher order tensors transforming, e.g., stresses into other stresses or strains into stresses. This also has to be considered for the special unit tensors $\boldsymbol{I}^{\text {vol }}, \boldsymbol{I}^{\text {dev }}$.

\subsection{Quasistatic Damage}

Damage is related to ongoing microcracking and stiffness degradation of quasi-brittle materials like concrete [8]. Isotropic quasistatic damage stress-strain behavior can be described with

$$
\begin{aligned}
& \boldsymbol{\sigma}^{v o l}=(1-D) 3 K_{0} \boldsymbol{\epsilon}^{v o l} \\
& \boldsymbol{\sigma}^{d e v}=(1-D) 2 G_{0} \boldsymbol{\epsilon}^{\text {dev }}
\end{aligned}
$$

with the initial Bulk modulus $K_{0}=E_{0} /(3(1-$ $2 \nu)$ ), the initial Young's modulus $E_{0}$, the Poission's ratio $\nu$, which is assumed as constant, and the initial shear modulus $G_{0}=E_{0} /(2(1+\nu))$. The parameter $0 \leq D \leq 1$ is the scalar damage parameter, which generally grows during a loading history according to an evolution law. A form

$$
D(\kappa)= \begin{cases}0 & \kappa \leq e_{0} \\ 1-\mathrm{e}^{-\left(\frac{\kappa-e_{0}}{e_{d}}\right)^{g_{d}}} & \kappa>e_{0}\end{cases}
$$

is chosen with material parameters $e_{0}, e_{d}, g_{d}$. Eq. (3) derives the damage parameter $D$ from an equivalent damage strain $\kappa$, which serves as state variable. Rates $\dot{D}, \dot{\kappa}$ of damage and equivalent damage strain are related by

$$
\dot{D}=\frac{\mathrm{d} D}{\mathrm{~d} \kappa} \dot{\kappa}
$$

A damage function $F=F(\epsilon, \kappa)$ connects the equivalent damage strain to the triaxial strain state. Damage grows during loading and remains constant during unloading. Both are ruled by Kuhn-Tucker conditions $F \leq 0, \dot{\kappa} \geq$ $0, F \dot{\kappa}=0$ leading to a consistency condition

$$
\dot{F}=\frac{\partial F}{\partial \boldsymbol{\epsilon}} \cdot \dot{\boldsymbol{\epsilon}}+\frac{\partial F}{\partial \kappa} \dot{\kappa}=0
$$

in case of loading. According to [6] a damage function

$F=c_{1} J_{2}+\kappa\left(c_{2} \sqrt{J_{2}}+c_{3} \epsilon_{1}+c_{4} I_{1}\right)-\kappa^{2}$

is chosen with material parameters $c_{1} \ldots c_{4}$, the largest principal strain $\epsilon_{1}$, the first strain invariant $I_{1}$ and the second invariant $J_{2}$ of the strain deviator. In case of loading the rate form of the stress-strain relation is given by

$$
\begin{aligned}
& \dot{\boldsymbol{\sigma}}^{v o l}=3 K_{0}\left[(1-D) \dot{\boldsymbol{\epsilon}}^{v o l}-\dot{D} \boldsymbol{\epsilon}^{v o l}\right] \\
& \dot{\boldsymbol{\sigma}}^{d e v}=2 G_{0}\left[(1-D) \dot{\boldsymbol{\epsilon}}^{d e v}-\dot{D} \boldsymbol{\epsilon}^{d e v}\right]
\end{aligned}
$$

With the values of $\boldsymbol{\epsilon}, \dot{\boldsymbol{\epsilon}}$ given, the values for $\kappa, \dot{\kappa}$ and $D, \dot{D}$ are determined through Eqs. (3.6). 


\subsection{Regularization and Retarded Damage}

Damage generally leads to a softening behavior of materials, i.e. stress values reach an absolute maximal value and then decrease with increasing absolute values of strain. This leads to localization phenomena with crack bands of finite width in concrete structures. Such crack bands mainly arise from the mesoscopic heterogeneity of concrete with the interaction of mortar and aggregates in a random distribution.

Softening constitutive laws in Finite Element Methods lead to a fundamental mesh dependency of results regarding the structural behavior in areas of softening or cracking, respectively. Several regularization approaches are proposed to overcome this deficiency [7], [11]. We basically consider gradient damage to introduce the concept of retarded damage. While in the basic damage approach the equivalent damage strain $\kappa$ directly depends on strain through the damage function $F$, see Eq. (6), gradient damage uses an equivalent gradient strain $\bar{\kappa}$ which is coupled to strain through

$$
\bar{\kappa}(\boldsymbol{x})-c \Delta \bar{\kappa}(\boldsymbol{x})=\kappa(\boldsymbol{x}), \quad c=\frac{R^{2}}{2}
$$

with the spatial coordinate $\boldsymbol{x}$, the Laplace differential operator

$$
\Delta \bar{\kappa}(\boldsymbol{x})=\frac{\partial^{2} \bar{\kappa}}{\partial x^{2}}+\frac{\partial^{2} \bar{\kappa}}{\partial y^{2}}+\frac{\partial^{2} \bar{\kappa}}{\partial z^{2}}
$$

and a characteristic length $R$ as a measure for the macroscopic heterogeneity of the material. $R$ is assumed as a material parameter. High gradients of the strain leading to high gradients of $\kappa$ are transformed to a lower gradient course of $\bar{\kappa}$ due to Eq. (8). In particular, a coupling of this equation with a field of strains $\epsilon$ embedded in a finite element computation results in a field $\bar{\kappa}$ which is not mesh dependent anymore. Thus, the equivalent gradient strain $\bar{\kappa}$ is used to derive the scalar damage parameter $D$ in Eq. (3) instead of $\kappa$. This overcomes the mesh dependency and may provide a correct resolution of a crack band within the framework of macroscopic continuum mechanics.
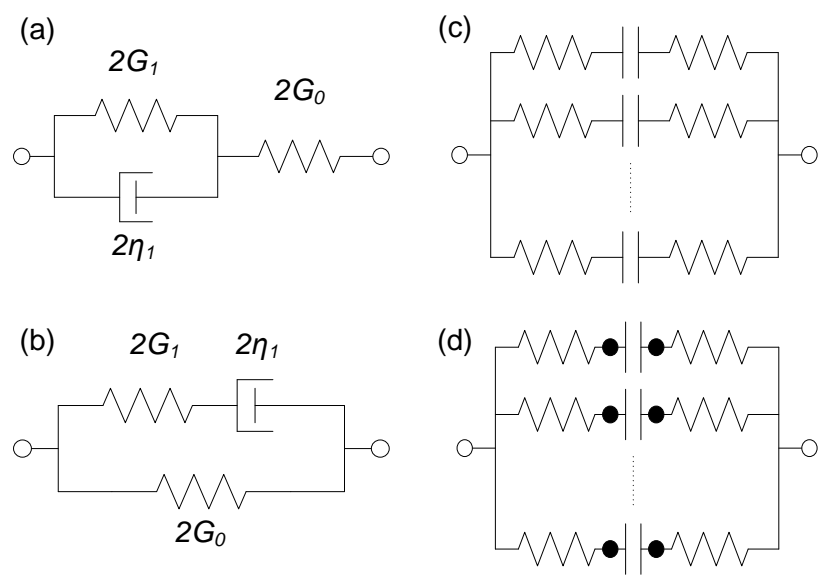

Figure 2: Rheologic models for a,b) viscoelasticity, c) damage, d) retarded damage.

Damage is a model for micro-cracking with the evolution of micro cracks and a microscopic movement of crack surfaces relative to their immediate surroundings. This should lead to inertial effects under high strain-rate conditions. We state the hypothesis that damage is retarded if a deformation is imposed fast. Thus, the wellknown approach Eq. (8) covering the spatial domain is extended in the time domain with

$a \ddot{\bar{\kappa}}(\boldsymbol{x})+b \dot{\bar{\kappa}}(\boldsymbol{x})+\bar{\kappa}(\boldsymbol{x})=\kappa(\boldsymbol{x})+c \Delta \bar{\kappa}(\boldsymbol{x})(10)$

with an inertial-like term $a \ddot{\bar{\kappa}}(\boldsymbol{x})$ and a dampinglike term $b \dot{\bar{\kappa}}(\boldsymbol{x})$ as a first approach to model retarded damage on the macroscopic scale. Eq. (10) corresponds to a one-degree damped oscillator coupled to its surrounding through the term $\kappa(\boldsymbol{x})+c \Delta \bar{\kappa}(\boldsymbol{x})$. The material-like parameters $a, b$ will be discussed later. Rheological models for damage and retarded damage are shown in Fig. 2k, d. Material parameters assigned to stiffnesses, strengths and masses of single elements are stochastic in such models.

\subsection{Damaged Viscoelasticity}

Regarding concrete viscosity arises from the resistance of a mortar's microstructure activated under high strain-rate conditions by the movement of more or less bound water in the mortar's capillary systems [5]. Deviatoric viscoelastic three-parameter models ( [10]) are considered in a first approach, see Fig. 2. 
Model (a) combines a Kelvin-Voigt element and a spring in series, model (b) a Maxwell element and a spring in parallel. Their behavior is ruled by ordinary differential equations of first order applied to the deviatoric components

$$
\dot{\boldsymbol{\sigma}}^{d e v}=q_{1} \dot{\boldsymbol{\epsilon}}^{d e v}+q_{0} \boldsymbol{\epsilon}^{d e v}-p_{0} \boldsymbol{\sigma}^{d e v}
$$

Model (b) activates a stiffness $G_{1}$ in addition to the quasistatic stiffness $G_{0}$ for high strain-rates depending on the viscosity $\eta_{1}$. It belongs to the class of generalized Maxwell models and will be used in the following. Its strain-rate is determined with

$$
\begin{aligned}
\dot{\boldsymbol{\epsilon}}^{d e v}= & \frac{1}{2 G_{1}}\left(\dot{\boldsymbol{\sigma}}^{d e v}-2 G_{0} \dot{\boldsymbol{\epsilon}}^{d e v}\right) \\
& +\frac{1}{2 \eta_{1}}\left(\boldsymbol{\sigma}^{d e v}-2 G_{0} \boldsymbol{\epsilon}^{d e v}\right)
\end{aligned}
$$

composed of a viscous part and an elastic part of the Maxwell-element (subscript 1). Strain (without subscript) of the spring-element (subscript 0) and the Maxwell-element are the same as they are considered to act in parallel. A comparison of Eqs. (11/2) leads to

$$
\begin{gathered}
q_{1}=2\left(G_{0}+G_{1}\right)=2 G_{0}\left(1+\frac{1}{\varphi}\right) \\
q_{0}=\frac{2 G_{0} G_{1}}{\eta_{1}}=\frac{2 G_{0}}{\zeta} \\
p_{0}=\frac{G_{1}}{\eta_{1}}=\frac{1}{\zeta}
\end{gathered}
$$

with a creep number $\varphi=G_{0} / G_{1}$ and a relaxation time $\zeta=\eta_{1} / G_{1}$. This yields an initial modulus $G_{1}+G_{0}$ under instantaneously applied loading and a final Modulus $G_{0}$. A low creep number leads to high initial stiffness and a high creep number to low initial stiffness. The relaxation time $\zeta$ rules the speed of creep. With a low $\zeta$ creep is fast, with a high $\zeta$ creep is slow. The non-viscous case has $\zeta \rightarrow 0$ and $\boldsymbol{\sigma}^{d e v} \rightarrow(1-D) 2 G_{0} \boldsymbol{\epsilon}^{d e v}$.

The approach Eq. (12) remains to be modified with respect to damage. We choose

$$
\begin{aligned}
\dot{\boldsymbol{\epsilon}}^{d e v}= & \frac{1}{(1-D) 2 G_{1}}\left[\dot{\boldsymbol{\sigma}}^{d e v}-(1-D) 2 G_{0} \dot{\boldsymbol{\epsilon}}^{d e v}\right. \\
& \left.+\dot{D} 2\left(G_{0}+G_{1}\right) \boldsymbol{\epsilon}^{d e v}\right] \\
& +\frac{1}{2 \eta_{1}}\left[\boldsymbol{\sigma}^{d e v}-(1-D) 2 G_{0} \boldsymbol{\epsilon}^{d e v}\right]
\end{aligned}
$$

corresponding to a common damage of both springs whereby each is ruled by Eqs. (27).
This introduces $\dot{D} 2\left(G_{0}+G_{1}\right) \boldsymbol{\epsilon}^{\text {dev }}$ as additional contribution to strains due to elastic degradation and leads to

$$
\begin{aligned}
& \dot{\boldsymbol{\sigma}}^{d e v}=(1-D)\left[q_{1} \dot{\boldsymbol{\epsilon}}^{d e v}\right. \\
& \left.+(1-D) q_{0} \boldsymbol{\epsilon}^{d e v}-p_{0} \boldsymbol{\sigma}^{d e v}\right]-\dot{D} q_{1} \boldsymbol{\epsilon}^{d e v}
\end{aligned}
$$

Regarding $\dot{\boldsymbol{\sigma}}=\dot{\boldsymbol{\sigma}}^{\text {vol }}+\dot{\boldsymbol{\sigma}}^{\text {dev }}$ the Eqs. (7), 15 are combined to yield

$$
\begin{aligned}
& \dot{\boldsymbol{\sigma}}=(1-D) \boldsymbol{E}_{1} \cdot \dot{\boldsymbol{\epsilon}}-\dot{D} \boldsymbol{\sigma}_{1} \\
& +(1-D)\left[(1-D) q_{0} \boldsymbol{\epsilon}^{d e v}-p_{0} \boldsymbol{\sigma}^{d e v}\right]
\end{aligned}
$$

with

$$
\begin{aligned}
& \boldsymbol{\sigma}_{1}=\boldsymbol{E}_{1} \cdot \boldsymbol{\epsilon} \\
\boldsymbol{E}_{1}= & 3 K_{0} \boldsymbol{I}^{\text {vol }}+q_{1} \boldsymbol{I}^{\text {dev }}
\end{aligned}
$$

using Eq. (1). The non-viscous case has a creep number $\varphi \rightarrow \infty$ and the matrix $\boldsymbol{E}_{1}$ becomes the isotropic elasticity matrix $\boldsymbol{E}_{0}=3 K_{0} \boldsymbol{I}^{\text {vol }}+$ $2 G_{0} \boldsymbol{I}^{\text {dev }}$.

\subsection{Material Parameters}

The quasistatic part of the constitutive law and the meaning of its material parameters and calibration procedures have been discussed in [6]. An appropriate choice of parameters of Eqs. (36) for a common concrete C40 according to [2] is given in Table 11. The parameters $e_{0}, e_{d}, g_{d}$ rule uniaxial compressive behavior while the parameters $c_{1} \ldots c_{4}$ determine the relations of uniaxial tensile and bi- and triaxial compressive strength to uniaxial compressive strength.

Table 1: Parameters for quasistatic behavior(C40)

\begin{tabular}{ll}
\hline Young's modulus $E_{0}$ & $36000 \mathrm{MPa}$ \\
shear modulus $G_{0}$ & $15000 \mathrm{MPa}$ \\
Poisson's ratio $\nu_{0}$ & 0.20 \\
uniaxial compressive strength $f_{c}$ & $50 \mathrm{MPa}$ \\
uniaxial tensile strength $f_{c t}$ & $3.5 \mathrm{MPa}$ \\
\hline damage parameter $e_{0}$ & $-6.77 \cdot 10^{-6}$ \\
damage parameter $e_{d}$ & $3.25 \cdot 10^{-3}$ \\
damage parameter $g_{d}$ & 2.0 \\
\hline damage parameter $c_{1}$ & 3.1819 \\
damage parameter $c_{2}$ & -0.3419 \\
damage parameter $c_{3}$ & 11.7710 \\
damage parameter $c_{4}$ & 4.4077 \\
\hline
\end{tabular}


Additional material parameters have been introduced with $a, b$ for retarded damage and $\varphi, \zeta$ for viscosity under high strain-rate conditions. As the dynamic strength increase factor is different for tension and compression it is appropriate to choose different values depending on a measure indicating a range between tensile and compressive states. The tension indicator $-1 \leq \xi \leq 1$ is chosen for this purpose. A value $\xi=1$ indicates states on the tensile meridian of principal strains, $x=-1$ states on the compressive meridian. Transition states are interpolated. Regarding, e.g., the creep number its value $\varphi$ is determined from

$$
\varphi=\frac{1}{2}\left[(1-\xi) \varphi_{c}+(1+\xi) \varphi_{t}\right]
$$

with a constant creep number $\varphi_{c}$ for purely compressive states and a constant creep number $\varphi_{t}$ for purely tensile states. Analogous relations are used for the relaxation time $\zeta$ and for damage inertia $a$ and damping $b$. The particular values are chosen in order to reproduce uniaxial dynamic increase factors as shown in Fig. 1 (assuming the intrinsic material response, i.e. the stress corrected by additional inertia terms, as shown e.g. in [3]) and are listed in Table 2. In a first estimation these values should be independent from concrete grading for normal graded concretes.

Table 2: Parameters for strain-rate sensitivity

\begin{tabular}{ll}
\hline creep number tension $\varphi_{t}$ & 1.0 \\
creep number compression $\varphi_{c}$ & 5.0 \\
relaxation time tension $\zeta_{t}$ & $5 \mathrm{~ms}$ \\
relaxation time compression $\zeta_{c}$ & $1 \mathrm{~ms}$ \\
\hline damage inertia tension $a_{t}$ & $5 \cdot 10^{-5} \mathrm{~ms}^{2}$ \\
damage inertia compression $a_{c}$ & $1 \cdot 10^{-5} \mathrm{~ms}^{2}$ \\
damage damping tension $b_{t}$ & $1 \cdot 10^{-3} \mathrm{~ms}$ \\
damage damping compression $b_{c}$ & $1 \cdot 10^{-3} \mathrm{~ms}$ \\
\hline
\end{tabular}

Fully triaxial simulations may be performed with each of these model variations whereby biaxial and uniaxial states are given as special cases.

\subsection{Implementation}

In a first approach the term $c \Delta \bar{\kappa}(\boldsymbol{x})$ will not be considered to make the constitutive law usable for the commercial explicit codes like, e.g., LS-Dyna through a user interface. This seems to thwart the initial regularization approach Eq. (8). Thus, convergence studies with different discretizations show that the reduced Eq. (10) together with the viscous approach might provide regularization properties. The discussion of a corresponding parameter study exceeds the scope ot this contribution. Formulation of user-defined Finite-Element types for, e.g., LS-Dyna including the nonlocal equivalent gradient strain $\bar{\kappa}$ as a further field variable and its influence on the simulation has to be postponed to future work.

The explicit LS-Dyna is actually used as solver for the following simulations with the current material law for concrete implemented as a user defined material. This allows to use proven methods for time step selection, large displacements and contact algorithms. Furthermore, LS-Dyna provides extensive libraries for standard element types and common materials. 3-D hexahedral elements with eight nodes are chosen for spatial discretization, furthermore bronze and aluminum materials for projectiles and incident and transmitter bars of Split-Hopkinson-Bar experimental setups discussed in the following.

\section{HOMOGENEOUS BEHAVIOR}

Homogeneous material behavior is simulated with LS-Dyna with the material model as has been described in Section 2 implemented through a user interface. A one-element set-up is used. The edge length of $1 \mathrm{~mm}$ was chosen relatively short in order to minimize structural inertia effects. Uniaxial stress states with desired strain-rates are generated by prescribed displacement of appropriate element nodes.

\subsection{Uniaxial Compressive Behavior}

The resulting stress-strain relations in compression are given in Fig. 3 depending on a range of strain-rates. Quasistatic stress states 
are supposed to arise for strain-rates with $10^{-5} 1 / \mathrm{s}$. The quasistatic stress-strain curve with a strength of $50 \mathrm{MPa}$ at a strain of $2.3 \%$ serves as a reference. The application of higher strain-rates shows increasing maximum stresses or strength, respectively, with increasing associated strains, see Fig. 3. The corresponding DIF-values up to roughly 3 basically agree with experimental evidence, see Fig. 1. An increased initial Young's modulus is computed with higher strain-rates. Available experimental data about this effect are not unambiguous. Furthermore, the changing shape of stress-strain relations compared to the quasistatic curve needs more investigations.

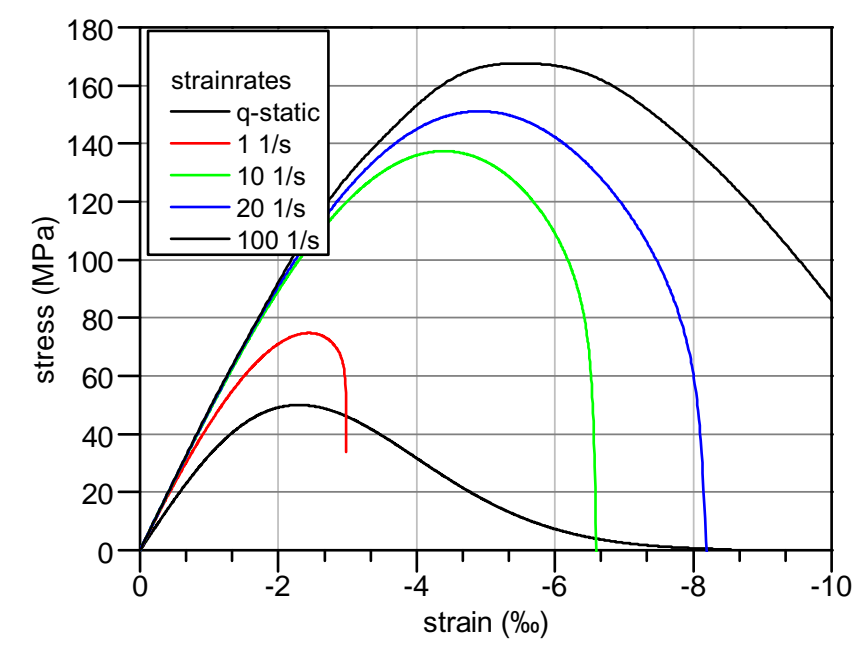

Figure 3: Stress-strain relations for uniaxial compression.

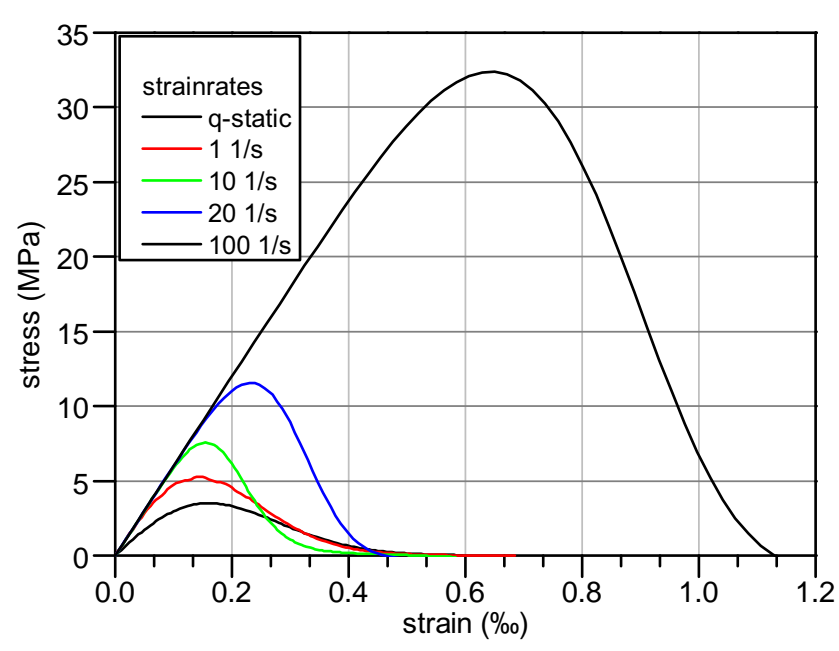

Figure 4: Stress-strain relations for uniaxial tension.

\subsection{Uniaxial Tensile Behavior}

Computed stress-strain relations for tension are shown in Fig. 4. Maximum stress values or strength again have to be compared to experimental DIF-values, see Fig. 1. The latter can be approximated by a bilinear curve in the tensile range. DIFs of up to 2 at a strain rate of about $101 / \mathrm{s}$ can be approximated by a first flat branch, while strain-rates above $10 \mathrm{1} / \mathrm{s}$ and up to $1001 / \mathrm{s}$ can be approximated by a second steeper branch with DIFs of up to 10. The computed results show a good agreement to this characteristic. Similar to compression the initial Young's modulus again increases, but the change of shape in the stress-strain relations is less pronounced.

\subsection{Biaxial Behavior}

For further examinations of the strain-rate dependent material model a number of simulations were performed under biaxial loading conditions. Prescribed nodal velocities are now applied in two directions, whereby the velocity of the secondary direction is scaled down to a fraction of the main direction velocity. Uniaxial strength as has been computed before is a special case with the corresponding values lying on the coordinate axes. The computed quasistatic biaxial strength, which is the most inner closed curve, see Fig. 5, again serves as a reference. Its values resemble the experimentally determined quasistatic biaxial failure envelope to a good extent.

Investigations of biaxial strength of concrete depending on strain-rates are rare up to now. Experimental data basically is not available. Nevertheless, the material model allows for a simulation. The results should be viewed as a first approach as they are an outcome of material parameters calibrated to reproduce certain aspects of uniaxial concrete behavior.

Fig. 5 shows computed maximum biaxial stresses. Closed strength curves are parametrized by the primary strain-rate. The data of a single curve contains points sharing a constant strain-rate in the primary direction while the ratio between the primary and the sec- 
ondary directions strain-rate varies between -1 and 1 in steps of 0.1 . Thus, the elements resultant strain-rate level differs for each calculation run, limiting the direct comparability of the connected curve points.

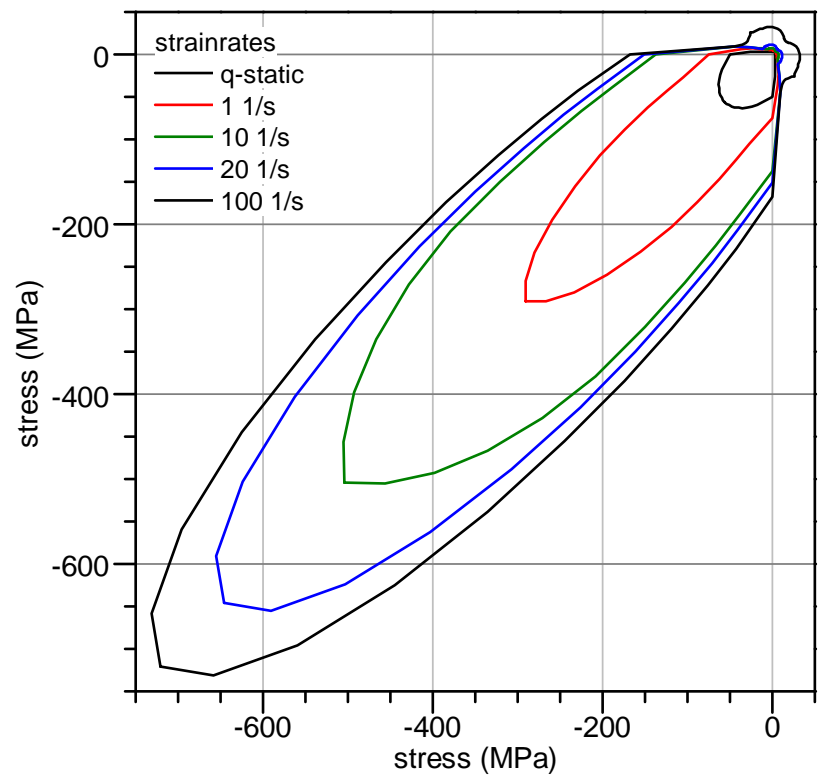

Figure 5: Biaxial dynamic failure curve.

It can be seen that biaxial strength increase may by far exceed the uniaxial strength increase in the purely compressive range, especially when both stress components have a similar level. This is not the case in the purely tensile range where uniaxial strength exceeds the biaxial values as well for quasistatic as for high strain-rate conditions.

The shape of the strength curves considerably differs in the area of combined tension and compression compared to pure compression or tension. This is presumably related with interpolation effects between purely compressive and purely tensile states according to the value of the tension indicator, see Eq. (18). This approach ensures on the one hand the continuity of the numerically determined biaxial failure envelope and on the other hand the distinction between moderate compressive and higher tensile strength increase. Another point to consider is the influence of the Poisson's ratio, which is assumed as constant. Uniaxial extension for ex- ample evokes tensile stresses in a first direction while shortening occurs in the second and third direction. Prescribed moderate compressive strain in the secondary direction counteracts the shortening induced by the Poisson's effect and cannot lead to compression as long as the tensile force dominates. A combination of both interpolation and Poisson's effects presumably superposes to the special shape of the strength curves in the upper corner of Fig. 5 .

\section{Biaxial Split-Hopkinson-Bar - Experi- ments}

Biaxial experimental results were obtained from a biaxial Split-Hopkinson-Bar (SHB) at the Institute of Concrete Structures at the Technische Universität Dresden.

\subsection{Experimental Setup}

The test setup is basically identical to the one shown in Fig. 6 .

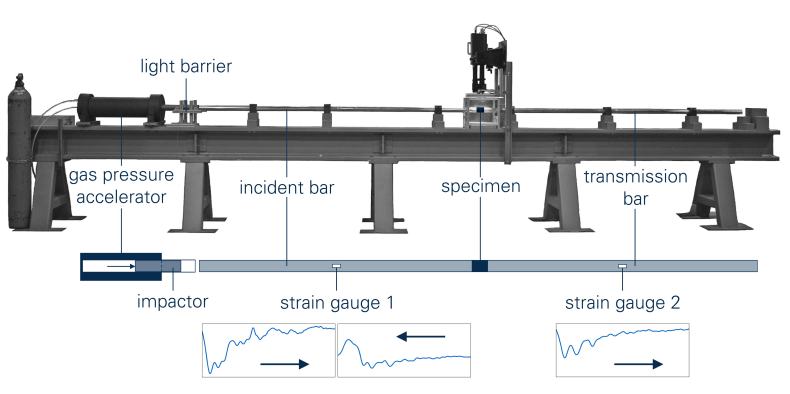

Figure 6: Split-Hopkinson-Bar basics.

It consists of a gas pressure accelerator with the impactor and the specimen sandwiched between the incident and the transmission bar, both made of aluminum and each with a diameter of $50 \mathrm{~mm}$ and a length $2.78 \mathrm{~m}$. The gas pressure accelerator can be charged with up to 10 bar compressed air and speed up the bronze impactor $(\mathrm{d}=49.5 \mathrm{~mm} ; \mathrm{l}=120 \mathrm{~mm} ; \mathrm{m}=2040 \mathrm{~g})$ to an impact velocity of 10 to $30 \mathrm{~m} / \mathrm{s}$. This impact induces a compressive impulse, which propagates through the incident bar with a wave propagation velocity of approx. $5000 \mathrm{~m} / \mathrm{s}$. The compressive impulse is measured in the middle of the incident bar by strain gauges. 
At the end of the incident bar the impulse reaches the interface between aluminum and specimen. Here the impulse is partly reflected due to the difference of impedance between aluminum and concrete and partly transmitted into the specimen. The reflected part propagates as tension wave in reversed direction through the incident bar and is measured again in the middle of the incident bar.

The part transmitted into the specimen generally reaches the concrete strength and leads to failure. Nevertheless, the transmitted part propagates through the specimen and might change its amplitude and shape due to the nonlinear concrete behavior. Further on, it is transmitted as compression wave into the transmission bar where it is finally gauged again in the middle of the bar.

The biaxial test configuration shown in Fig. 7 consists of two perpendicular Split-Hopkinson-Bars with all four rods ending in one middle point where the specimen is located. To get synchronized impulses in both axes several additional sensors, controllers and valves are necessary to control the impactor as precise as possible.

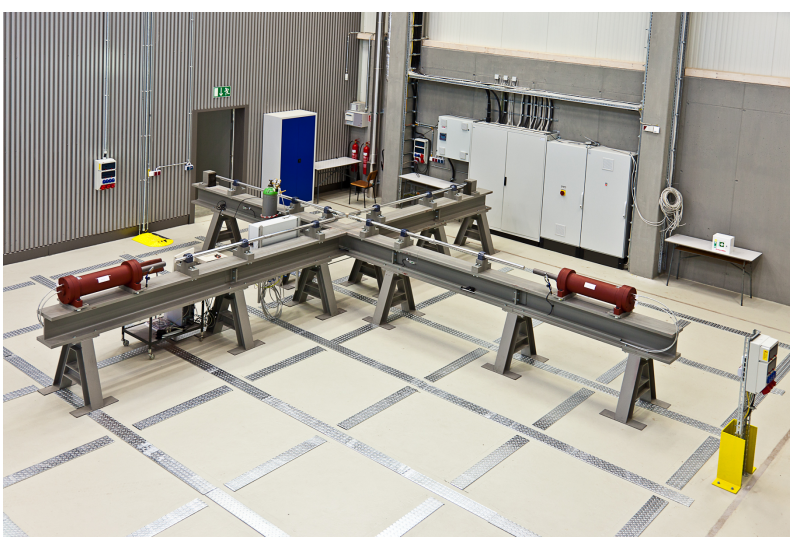

Figure 7: Biaxial Split-Hopkinson-Bar.

The examined concrete has a uniaxial static compression strength of $40.4 \mathrm{MPa}$ determined at cylindrical specimen $(\mathrm{d}=150 \mathrm{~mm}$; $1=300 \mathrm{~mm}$ ). The specimens for the biaxial tests are cubes with an edge length of $60 \mathrm{~mm}$.

\subsection{Basic theory}

Three single strain pulses, namely incident wave $\left(\epsilon_{i}\right)$, reflected wave $\left(\epsilon_{r}\right)$ and transmitted wave $\left(\epsilon_{t}\right)$ can be extracted from the measured strain data for every direction. According to the three-wave-analysis ( [3] ) the specimen's stress $\left(\sigma_{s}\right)$, strain-rate $\left(\dot{\epsilon}_{s}\right)$ and strain $\left(\epsilon_{s}\right)$ in a first approach can be calculated with with

$$
\begin{gathered}
\sigma_{s}(t)=\frac{E_{b} \cdot A_{b}}{2 \cdot A_{s}} \cdot\left(\epsilon_{i}(t)+\epsilon_{r}(t)+\epsilon_{t}(t)\right) \\
\dot{\epsilon}_{s}(t)=-\frac{c_{b}}{l_{s}} \cdot\left(-\epsilon_{i}(t)+\epsilon_{r}(t)+\epsilon_{t}(t)\right) \\
\epsilon_{s}(t)=\int \dot{\epsilon}_{s}(t) \cdot d t
\end{gathered}
$$

with the wave propagation velocity $c_{b}$ and the modulus of elasticity $E_{b}$ of the bars and the cross sectional area of the bars $A_{b}$ and the specimen $A_{s}$. These approaches are anlaytically derived under the assumption of basically linear specimen behavior and uniaxial states uncoupled in both directions.

\subsection{Test Procedure}

In the biaxial experiment the cubic specimen is centered between the four aluminum rods with a projecting of minimum $5 \mathrm{~mm}$ at all sides. A layer of molybdenum sulfide is applied on the surface of the specimen. Two experimental samples are exemplarily described. The impactors of the first sample have an impact speed of 23.5 and $23.0 \mathrm{~m} / \mathrm{s}$ in axis one and two. The two incident waves in axis one and two have a time difference of $0.010 \mathrm{~ms}$. In the second sample the impactor speeds are 23.6 and $23.1 \mathrm{~m} / \mathrm{s}$ and the time delay is $0.285 \mathrm{~ms}$.

\section{Biaxial Split-Hopkinson-Bar - Simula- tion}

The simulation is again performed with L-Dyna with a user interface of the material model. Meshing uses quad elements for all parts. Edge length of elements is $5 \mathrm{~mm}$ for the incident and transmission bars as well as for the 
impactor. A finer mesh was used for the specimen with an element size of approx. $2.5 \mathrm{~mm}$. The material behavior of the impactor and the bars was assumed linear elastic, whereas for the concrete specimen the nonlinear strain-rate dependent model was assumed. Simple contact algorithms were used allowing compressive loads to be transferred between slave nodes and master segments. The friction coefficient was found to be essential for the calculation results quality and was set to 0.45 between the concrete and aluminum parts.

\subsection{Model setup}

The biaxial Split-Hopkinson-Bar dimensions used for the study correspond to the experimental setup described in Section 4. In all cases both impactors are given an initial velocity of $20 \mathrm{~m} / \mathrm{s}$, accounting for inaccuracies in measurement. The experimentally measured time difference between the two waves is numerically incorporated as unequal distance between the incident bar and the impactor for the two axes. In detail a distance of 0.2 respectively $5.7 \mathrm{~mm}$ results in a delay of the time of 0.010 respectively $0.285 \mathrm{~ms}$ of first contact between the impactor and incident bar in the second axis.

\subsection{Strain Waves}

Fig. 8 and 9 compare the experimentally measured with the numerically calculated strain waves recorded at the strain gauge positions in case of a time delay of $0.010 \mathrm{~ms}$. Both computed incident waves are in good accordance with the experimental ones and the computed maximal transmitted strain agrees with the experimental data. The friction coefficient of 0.45 between concrete and aluminum is of prime importance at this point, as it reduces the strain of the transmitted wave about $25 \%$, compared to the case with friction coefficient set to 0 . However, the reflected wave comes with differing strain levels between experiment and modelling. A possible cause may be the prompt brittle failure of the first concrete elements in contact with the incident bar, forming a free end in terms of reflection. Another point on which experiment and modelling do not coincide is the wave propagation velocity in the specimen, which causes the numerical pulse to arrive earlier at the strain gauge position than the experimental one. Remarkable is also the fastly declining softening behaviour of concrete in case of the computation results.

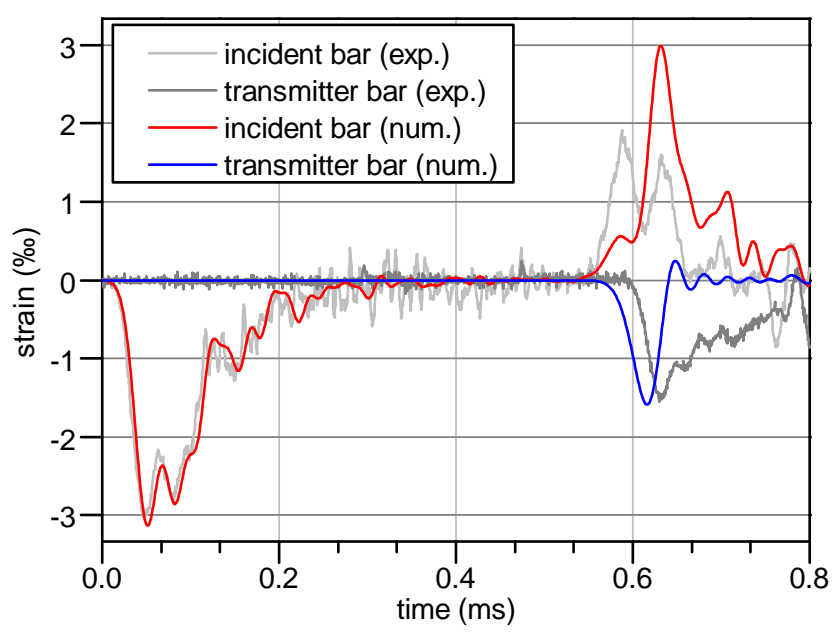

Figure 8: Strain waves for $0.010 \mathrm{~ms}$ offset: first axis.

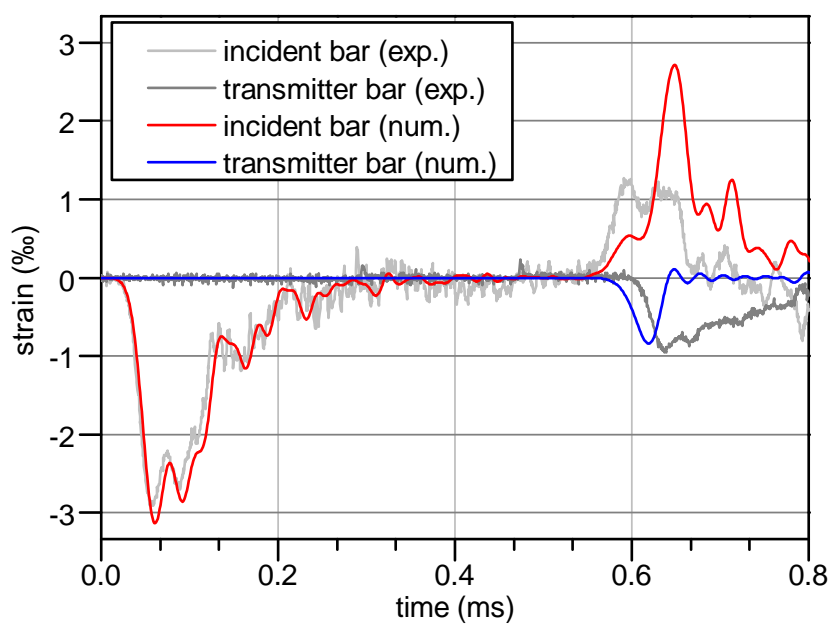

Figure 9: Strain waves for 0.010 ms offset: second axis.

\subsection{Global Results}

\subsubsection{Stress-Strain Relations}

Global stress-strain curves were determined both for the experiment and the calculation in accordance to the three-wave-analysis described in Section 4.2 They are shown in Fig. 10 and in general indicate an accordance. 


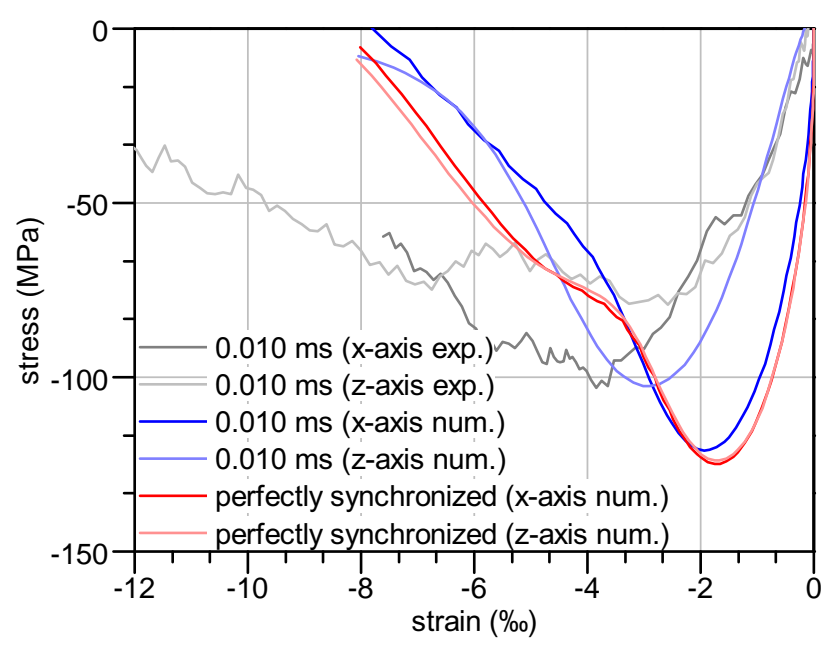

Figure 10: Experimental and numerical global results

\subsubsection{Crack pattern}

Fig. 11 compares the observed crack patterns with the spatial damage distribution in the specimen and how they are influenced by the time delay between the two pulses.
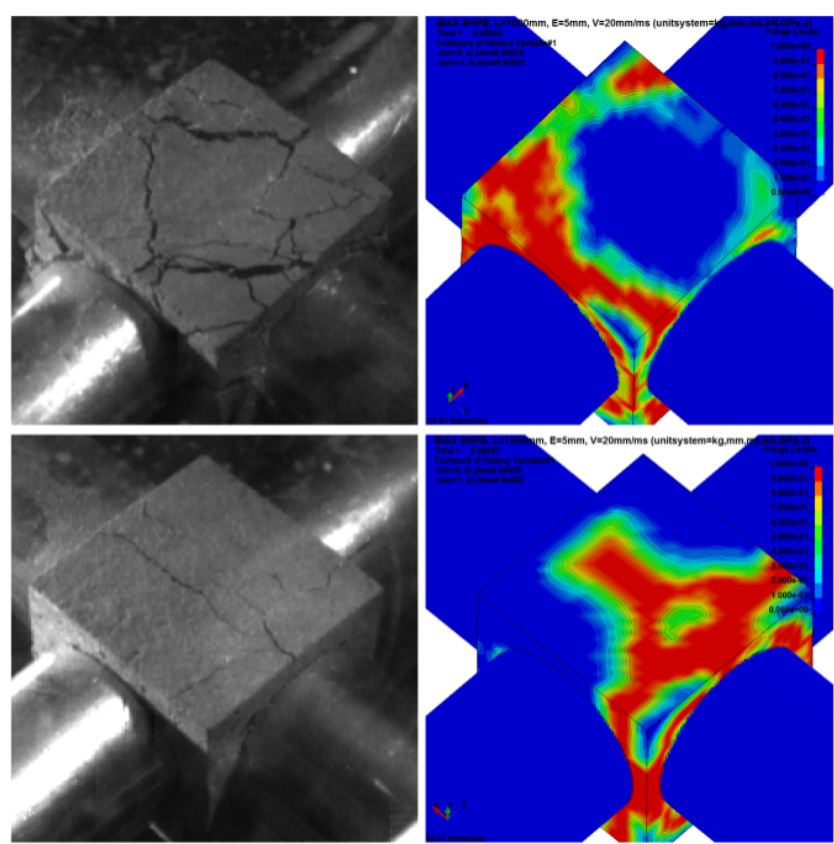

Figure 11: Crack pattern and damage distribution for a time delay of $0.010 \mathrm{~ms}$ (above) and $0.285 \mathrm{~ms}$ (below).

The upper scenario accounts for a time delay of $0.010 \mathrm{~ms}$ and the lower scenario for a time delay of $0.285 \mathrm{~ms}$. In the first case the load causes cracks to form diagonally pointing towards the middle of the adjacent aluminum bars, which arises in a rhombic pattern. The bigger time difference, in contrast, leads cracks to open parallel to the first impulse direction. Fine cracks can also be detected right-angled to that close to the load application point, probably being influenced by prevented lateral strain.

\subsection{Local Results}

The numerical simulation allows for a comprehensive evaluation of stresses and strains in every element during the whole time. This leads to local results which are discussed in the following.
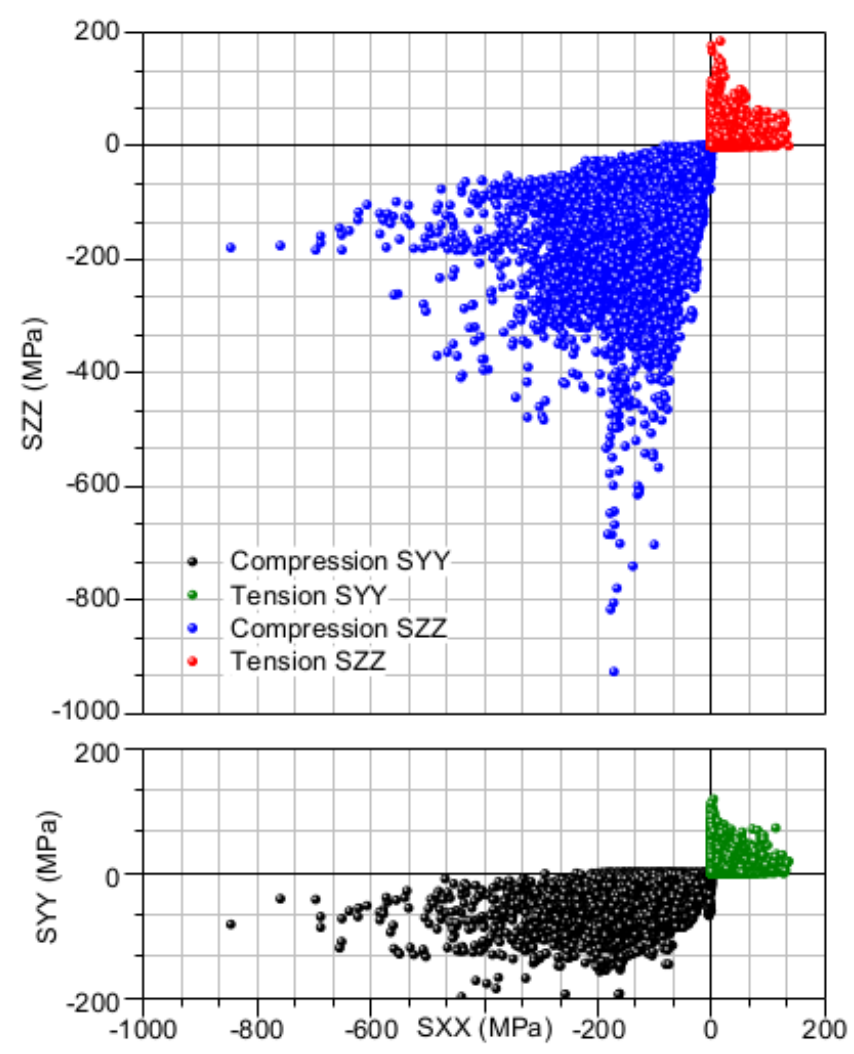

Figure 12: Triaxial principle stresses for perfectly biaxial loading condition.

\subsubsection{Homogeneity}

The following results are computed with the assumption of perfectly synchronized impulses with equal amplitudes. This leads to identical stresses in the $z$-and $x$-direction. Fig. 12 indicates the extremal stresses reached for representative elements during the whole loading 
history. The upper part shows in-plane stress components $\sigma_{z z}$ (with the same stress components $\sigma_{x x}$ ), the lower part collateral stresses $\sigma_{y y}$. The $x-z$-plane spans the plane of the bar directions. These results indicate that assumptions about homogeneity of stresses and plane stress states are by far not fulfilled. This puts the applicability of Eqs. (19) - (21) into perspective.

\subsubsection{Variations of Local Results}

Fig. 13 shows local stresses for the upper specimen in Fig. 11. Each of the curves is related to a single element and indicates the in-plane stresses varying during the load history.

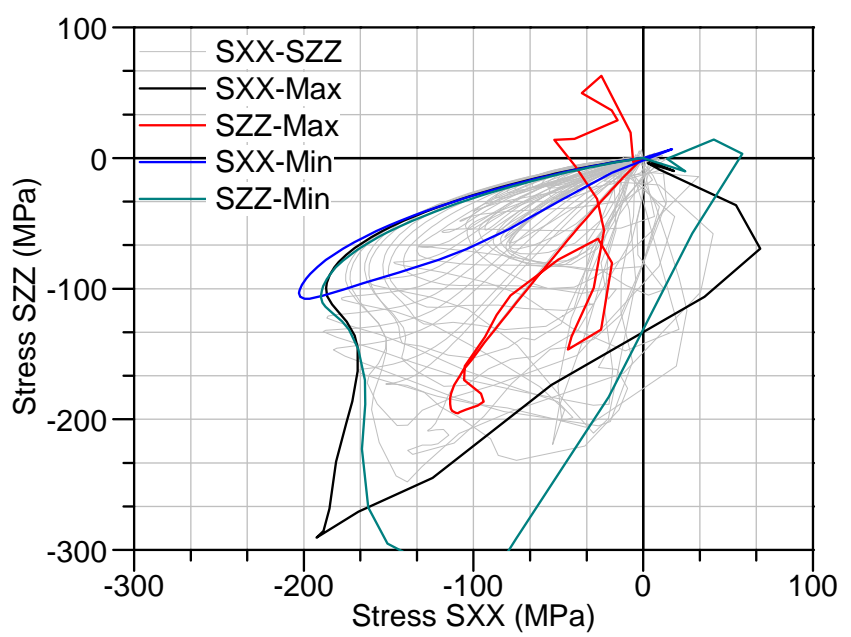

Figure 13: Local in-plane stress states varying with time.

The course of all stress states is shown in grey. The elements with the minimal and maximal values for each direction are highlighted with colors. The shown results indicate a complex stress history with sequences of loading, unloading and reloading.

Fig. 14 shows the corresponding stress-strain relations. Again elements with extremal values are highlighted with colors and the particular stress-strain relations can be considered as an indicator for failure and crack energy. The curves marked as SZZ-Min and SXX-Max remain straight indicating an nearly linear elastic path. SZZ-Min reaches a very high compressive stress level without failure which is probably caused by the more pronounced biaxial or triaxial stress state in the specific element. The blue curve for SXX-Min shows failure in the compressive domain with large compressive strain. The red SZZ-Max curve instead shows a first maximum in compression with unloading and later tensile failure at a lower tensile stress level.

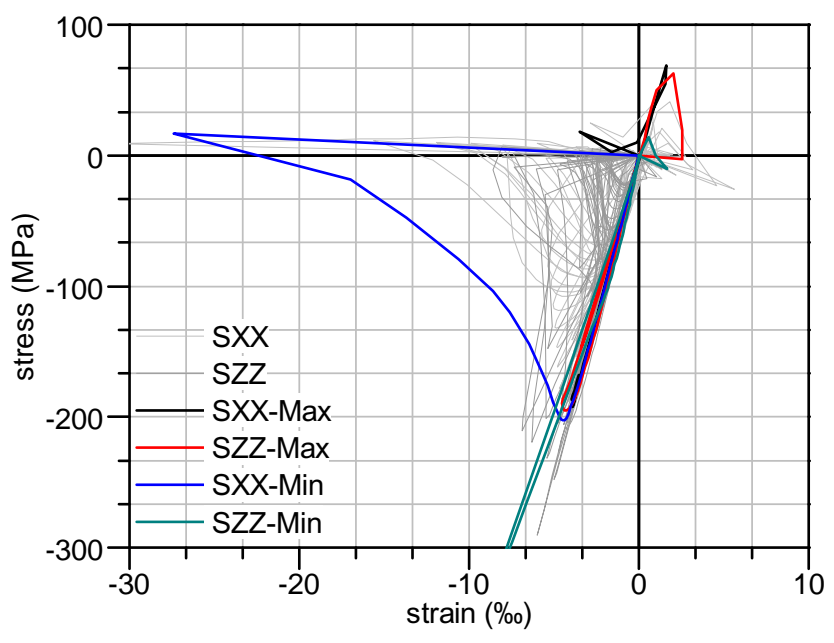

Figure 14: Local stress-strain relations.

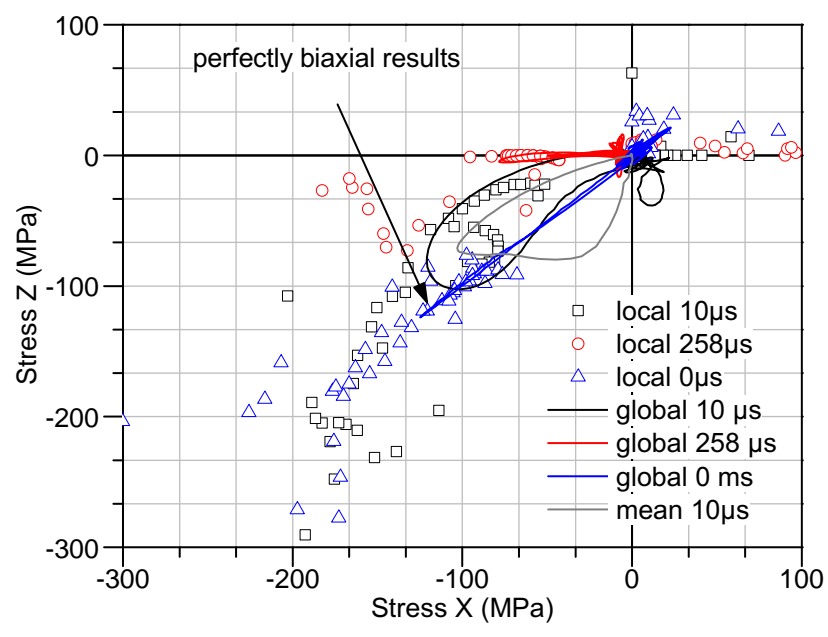

Figure 15: Local and global in-plane stresses.

Fig. 15 first of all shows local results of the elements with the extremal values in the tensile and compressive domain as single markers. Colors correspond to the previous figures indicating the different stress components. Different markers of the same color indicate different times. Furthermore, global results derived using 
Eqs. (19) - 21) are shown as curves or paths, respectively. Time varies along a path. The perfectly biaxial path - no time delay - appears as a straight line whereas the local maxima are scattered. All global values or paths underestimate stresses compared to the locally observed extrema. The dark-grey curve indicates an average of all considered elements, which is relatively close to the globally evaluated black one. This might confirm the global analysis procedure.

\section{CONCLUSIONS}

Experimental and simulation results were presented for concrete specimen exposed to biaxial loading under high strain-rate conditions. The simulation model gives a reasonable agreement with the experiments with some detail differences. Evaluated states are triaxial, spatially not homogeneous and highly variable in time in case of the biaxial Split-Hopkinson-Bar. Biaxial compressive strength by far seems to exceed unaxial strength, much more pronounced compared to quasistatic behavior. All these phenomena still need more investigations.

\section{ACKNOWLEDGEMENT}

The project is funded by the German Federal Ministry of Economic Affairs and Energy (BMWi, project no. 1501483 and 1501486) on basis of decision by the German Bundestag.

\section{REFERENCES}

[1] P. Bischoff and S. Perry. Compressive behavior of concrete at high strain rates. $M a-$ terials and Structures, 24:425-450, 1991.

[2] CEB-FIP. Model Code for Concrete Structures 2010. International Federation for Structural Concrete (FIB), Lausanne, Switzerland, 2012.

[3] W. W. Chen and B. Song. Split Hopkinson (Kolsky) bar : design, testing and applications. Springer, New York, NY ; Heidelberg [u.a.], 2011.

[4] J. Eibl and B. Schmidt-Hurtienne. Strainrate-sensitive constitutive law for con- crete. Journal of Engineering Mechanics, 125:1411-1420, 1999.

[5] E. J. Garboczi. Microstructure and transport properties of concrete. Performance Criteria for Concrete Durability, pages 198-212, 1995.

[6] U. Häussler-Combe and J. Hartig. Formulation and numerical implementation of a constitutive law for concrete with strainbased damage and plasticity. International Journal of Non-Linear Mechanics, 43:399-415, 2008.

[7] M. Jirasek. Nonlocal models for damage and fracture: comparison of approaches. International Journal of Solids and Structures, 35:4133-4155, 1998.

[8] J. Lemaitre and R. Desmorat. Engineering Damage Mechanics. Springer Verlag, Berlin, 2005.

[9] L. J. Malvar and C. A. Ross. Review of strain rate effects for concrete in tension. ACI Materials Journal, 95:735-739, 1998.

[10] L. E. Malvern. Introduction to the Mechanics of a Continuous Medium. Prentice-Hall, Englewood Cliffs, New Jersey, 1. auflage edition, 1969.

[11] R. Peerlings, R. de Borst, W. Brekelmans, and J. de Vree. Gradient enhanced damage for quasi-brittle materials. Int. J. Numer. Meth. Engng., 39:3391-3403, 1996.

[12] H. W. Reinhardt and J. Weerheijm. Tensile fracture of concrete at high loading rates taking account of inertiaand crack velocity effects. International Journal of Fracture, 51:31-42, 1991.

[13] P. Rossi. A physical phenomenon which can explain the mechanical behaviour of concrete under high strain rates. Materials and Structures, 24:422-424, 1991. 